\title{
BMJ Open Injury of the mammillothalamic tract in patients with subarachnoid haemorrhage: a retrospective diffusion tensor imaging study
}

\author{
Sung Ho Jang, ${ }^{1}$ Byung Yeon Choi, ${ }^{2}$ Seong Ho Kim, ${ }^{2}$ Chul Hoon Chang, ${ }^{2}$ \\ Young Jin Jung, ${ }^{2}$ Hyeok Gyu Kwon ${ }^{1}$
}

To cite: Jang SH, Choi BY, Kim SH, et al. Injury of the mammillothalamic tract in patients with subarachnoid haemorrhage: a retrospective diffusion tensor imaging study. BMJ Open 2014;4: e005613. doi:10.1136/ bmjopen-2014-005613

- Prepublication history for this paper is available online. To view these files please visit the journal online (http://dx.doi.org/10.1136/ bmjopen-2014-005613).

Received 3 May 2014 Revised 6 July 2014 Accepted 8 July 2014
CrossMark

For numbered affiliations see end of article.

Correspondence to Hyeok Gyu Kwon; khg0715@hanmail.net

\section{ABSTRACT}

Objective: Few studies have reported on injury of the mammillothalamic tract (MTT) in patients with stroke. However, no study in patients with subarachnoid haemorrhage (SAH) has been reported. Using diffusion tensor tractography, we attempted to investigate injury of the MTT in patients with SAH.

Methods: We recruited 16 patients with SAH and 15 control participants. DTI was obtained at $5.7 \pm 1.5$ weeks after onset and reconstruction of the MTT was performed using the probabilistic tractography method. The fractional anisotropy (FA) value and tract number of the MTT and the Mini-Mental State Examination (MMSE) score were determined. Values of FA and tract volume showing a decrement of more than two SDs that of normal control were defined as abnormal.

Results: The FA value and tract volume in the patient group were significantly lower than those in the control group $(p<0.05)$. In addition, MMSE showed strong $(r=0.67, p=0.005)$ positive correlation with tract volume without correlation with FA. In the individual analysis, 16 MTTs of 32 MTTs in 16 patients showed abnormalities of the MTT in terms of the FA value, the tract volume or the presence of a reconstructed MTT. As a result, $10(62.5 \%)$ of 16 patients showed abnormality of the MTT in at least one hemisphere.

Conclusions: We found that patients with SAH showed injury of the MTT and this injury showed correlation with cognitive dysfunction.

\section{INTRODUCTION}

The mammillothalamic tract (MTT) connects the mammillary body and the anterior thalamus as a part of the Papez circuit. ${ }^{1}$ Owing to the anatomical characteristics of the MTT, thin, short and located deep within the brain, accurate estimation of the MTT has been difficult in the live human brain. However, diffusion tensor tractography (DTT), which is derived from diffusion tensor imaging (DTI), allows for reconstruction of the MTT in the human brain. ${ }^{2}$

\section{Strengths and limitations of this study}

- This is the first study on the injury of the mammillothalamic tract (MTT) in patients with subarachnoid haemorrhage (SAH) using diffusion tensor imaging.

- The main strength of our study is to find the relation between MTT injury and cognitive function with SAH.

- The limitation to our study is that fibre tracking of the MTT might be affected by an artefact, such as an aneurysmal clip. Therefore, the conduct of further studies to overcome this limitation would be necessary.

Therefore, accurate estimation of the MTT in terms of DTI parameters and threedimensional configuration is now possible. ${ }^{2}$ A few studies have reported on injury of the MTT in thalamic haemorrhage and thalamic infarct; however, so far, little is known about injury of the MTT. ${ }^{3} 4$

Subarachnoid haemorrhage (SAH) is extravasation of blood into the subarachnoid space accompanied by various neurological sequelae in terms of memory, executive function, language, motor function and cranial nerve function. ${ }^{5-16}$ In particular, memory deficit is known to be one of the most common sequelae of $\mathrm{SAH}^{7} 81011^{14}$ In the past, previous studies have suggested that the neurotoxic effects of blood, vasospasm or increased intracranial pressure are pathogenic mechanisms of neural sequelae following $\mathrm{SAH}^{5-7}{ }^{17-23}$ After introduction of DTI, among the neural tracts associated with memory, injuries in the fornix and cingulum were reported in patients with $\mathrm{SAH}^{24}$ However, no study on neural injury of the MTT in patients with SAH has been reported. In this study, we hypothesised that the MTT would be injured due to SAH. 
In this study, using DTT, we attempted to investigate injury of the MTT in patients with SAH.

\section{METHODS}

\section{Participants}

Among 55 patients, 39 were excluded due to the hydrocephalus (8 patients), intracerebral haemorrhage (14 patients) or intraventricular haemorrhage (17 patients). The remaining 16 patients (male: 9, female: 7, mean age: $51.6 \pm 13.3$ years, range: $34 \sim 70$ years) and 15 normal healthy control participants (male: 7 , female: 8 , mean age: $48.1 \pm 15.1$ years, range: $20 \sim 67$ years) with no previous history of neurological, physical or psychiatric illness were recruited for this study. Inclusion criteria for patients were as follows: (1) first ever stroke, (2) age 30 70 years, (3) haemorrhage in the subarachnoid space due to aneurysmal rupture confirmed by a neuroradiologist, (4) DTI was scanned at a chronic stage after onset and (5) no hydrocephalus, intracerebral haemorrhage or intraventricular haemorrhage was found. Severity of SAH was assessed according to the modified Fisher CT grade, World Federation of Neurosurgical Societies (WFNS) and Hijdra score. ${ }^{25-27}$ In patients who showed any lesion or artefact due to the clipping or coiling along or around the MTT, the pathway between the mammillary body and thalamus was excluded. This study was conducted retrospectively and the study protocol was approved by the Institutional Review Board of a university hospital (figure 1).

\section{Clinical evaluation}

Cognitive function was evaluated at the time of DTI scanning. The Mini-Mental State Examination (MMSE) was used for assessment of cognitive impairment. The reliability and validity of the MMSE have been well established. $^{28}$

\section{Diffusion tensor tractography}

A 6-channel head coil on a 1.5 T Philips Gyroscan Intera (Philips Ltd, Best, the Netherlands) with single-shot echo-planar imaging was used for acquisition of DTI data. For each of the 32 non-collinear diffusion sensitising gradients, we acquired 67 contiguous slices parallel to the anterior commissure-posterior commissure line. Imaging parameters were as follows: acquisition matrix $=96 \times 96$; reconstructed to matrix $=128 \times 128$; field of view $=221 \times 221 \mathrm{~mm}^{2} ; \quad \mathrm{TR}=10726 \mathrm{~ms} ; \mathrm{TE}=76 \mathrm{~ms} ;$ parallel imaging reduction factor (SENSE factor) $=2$; EPI factor $=49 ; \mathrm{b}=1000 \mathrm{~s} / \mathrm{mm}^{2} ; \mathrm{NEX}=1$; and a slice thickness

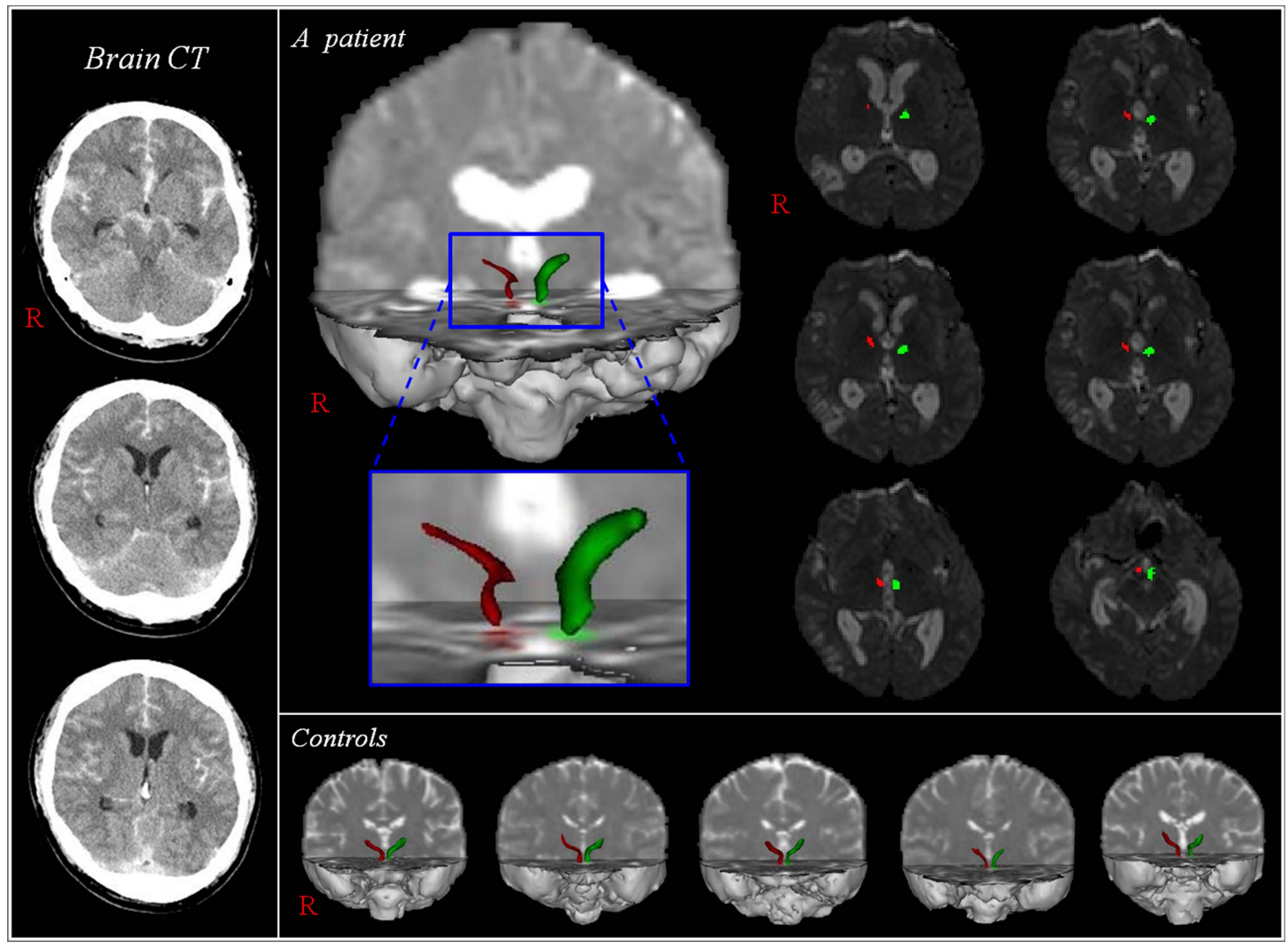

Figure 1 Diffusion tensor tractography for the mammillothalamic tract (MTT). Brain CT shows a subarachnoid haemorrhage and MTTs in both hemispheres of a patient (34-year-old man) are reconstructed between the mammillary body and the anterior thalamus. However, the right MTT (red) of the patient is thinned, compared with the left MTT (green) of the patients and normal control participants. 
of $2.3 \mathrm{~mm}$ (acquired voxel size $1.73 \times 1.73 \times 2.3 \mathrm{~mm}^{3}$ ). Affine multiscale two-dimensional registration using the Oxford Centre for Functional MRI of Brain (FMRIB) Software Library (FSL; http://www.fmrib.ox.ac.uk/fsl) was used for removal of eddy current-induced image distortions. ${ }^{29}$ Fibre tracking was performed using a probabilistic tractography method based on a multifibre model, and applied in this study utilising tractography routines implemented in FMRIB Diffusion (5000 streamline samples, $0.5 \mathrm{~mm}$ step lengths, curvature thresholds $=0.2) .{ }^{29-31}$ MTTs were determined by selection of fibres passing through three regions of interest (ROIs). Seed ROIs were placed on the mammillary body on the axial image. ${ }^{2}$ Two target ROIs were drawn at the portion of the MTT area (between the portion of the fornix and the red nucleus in the anteroposterior direction) at about the bicommissural level and the portion of the anterior thalamus on the axial image. ${ }^{2}$ Of 5000 samples generated from each seed voxel, results for each contact were the visualised threshold point at five streamline through each voxel for analysis. Values of fractional anisotropy (FA) and tract volume, which was determined by counting the voxels of MTT, were measured using MATLAB (Matlab R2007b, The Mathworks, Natick, Massachusetts, USA). Values of FA and tract volume showing a decrement of more than two SDs that of normal control were defined as abnormal.

\section{Statistical analysis}

We used SPSS software (V.15.0; SPSS, Chicago, Illinois, USA) for data analysis. Demographic data in duration from onset and age were tested for normality. Data on MTTs that were not reconstructed were excluded from the statistical analysis. An independent $t$ test was used for determination of variances in the value of FA and tract volume between the patient and control groups. Subsequently, using Pearson correlation, DTT parameters for FA and tract volume of the patient group were used in the determination of correlation with MMSE. ${ }^{32}$ The significant level of the $\mathrm{p}$ value was set at 0.05 .

\section{RESULTS}

A summary of the demographic data for the patient and control groups is shown in table 1 . The artery distribution of aneurysmal rupture for SAH in 16 patients was as follows: the anterior communicating artery: 11 patients $(68.8 \%)$, the middle cerebral artery: three patients $(18.8 \%)$, the posterior communicating artery: one patient $(6.2 \%)$ and the anterior cerebral artery: one patient $(6.2 \%)$. In addition, 12 patients $(75 \%)$ underwent aneurysmal clipping and two patients underwent endovascular coiling $(12.5 \%)$. The two remaining patients $(12.5 \%)$ received conservative treatment. The average MMSE was $19.8 \pm 8.8$. The average modified Fisher CT grade, WFNS and Hijdra score were 2.5 \pm 0.9 , $2.1 \pm 1.3$ and $15.7 \pm 6.3$, respectively. ${ }^{25-28}$ DTI scanning was performed at $5.7 \pm 1.5$ weeks (range: $4 \sim 8$ weeks) after

\begin{tabular}{|c|c|c|}
\hline & $\begin{array}{l}\text { Patient } \\
\text { group } \\
(n=16)\end{array}$ & $\begin{array}{l}\text { Control group } \\
(n=15)\end{array}$ \\
\hline Age (year) & $51.6 \pm 13.3$ & $48.1 \pm 15.1$ \\
\hline Sex, male/female & $9 / 7$ & $7 / 8$ \\
\hline Duration from onset (weeks) & $5.7 \pm 1.5$ & \\
\hline Modified Fisher grade & $2.5 \pm 0.9$ & \\
\hline WFNS & $2.1 \pm 1.3$ & \\
\hline Hijdra score & $15.7 \pm 6.3$ & \\
\hline $\begin{array}{l}\text { Ruptured artery } \\
\text { (ACoA:ACA:MCA:PCA) }\end{array}$ & $11: 1: 3: 1$ & \\
\hline $\begin{array}{l}\text { Operation type } \\
\text { (clipping:coiling:non) }\end{array}$ & $12: 2: 2$ & \\
\hline MMSE & $19.8 \pm 8.8$ & \\
\hline
\end{tabular}

SAH onset. Demographic data of the patient (duration from onset and age) and control (age) groups met the normality $(p>0.05)$ assumption.

A summary of the results of DTT parameters for MTT in the patient and control groups is shown in table 2 . Values for FA and tract volume were significantly lower in the patient group than in the control group $(p<0.05)$. The tract volume of the MTT showed a strong $(\mathrm{r}=0.67$, $\mathrm{p}=0.005$ ) positive correlation with MMSE; however, no correlation was observed between the FA value of MTT and MMSE $(\mathrm{r}=0.41, \mathrm{p}=0.11) .^{32}$

Figure 2 shows the results for incidence of injury of the MTT with DTT parameters. Among 32 hemispheres of 16 patients, the MTT was not reconstructed in five hemispheres of four patients (right hemisphere: two, left hemisphere: one, both hemispheres: one); in contrast, MTTs were reconstructed in all 30 hemispheres of 15 control participants. With regard to the FA and tract volume, nine MTTs in six patients (right hemisphere: one, left hemisphere: two, both hemispheres: three) and 13 MTTs in nine patients (right hemisphere: four, left hemisphere: one, both hemispheres: four) of 32 hemispheres in 16 patients revealed a decrement of more than two SDs, compared with control participants. Six MTTs in five patients (right hemisphere: three, left hemisphere: one, both hemispheres: one) showed a decrement of more than two SDs in FA and tract volume. As a result, 16 MTTs (right hemisphere: two, left hemisphere: two, both hemispheres: six) of 32 MTTs in 16 patients showed abnormalities of the MTT. Three patients in one hemisphere and one patient in both hemispheres whose MTT was not reconstructed belonged to these 16 patients. Consequently, in the individual analysis, the prevalence of MTT abnormality was $62.5 \%$ (10 of 16 patients). 
Table 2 Results of diffusion tensor tractography parameters of the mammillothalamic tract in the patient and control groups

\begin{tabular}{lllll}
\hline & Hemisphere & FA & p Value & Tract volume \\
\hline Patient group & Right & $0.36(0.04,0.36)$ & $0.015^{*}$ & $69.08(30.33,72.00)$ \\
& Left & $0.35(0.04,0.36)$ & & $78.86(28.80,80.50)$ \\
& Both & $0.36(0.04,0.36)$ & & $74.15(23.39,74.00)$ \\
Control group & Right & $0.38(0.04,0.38)$ & & $96.80(22.7,96.00)$ \\
& Left & $0.37(0.03,0.38)$ & & $99.73(23.8,103.00)$ \\
& Both & $0.38(0.03,0.38)$ & & $98.27(22.9,98.50)$ \\
\hline
\end{tabular}

Values represent mean $( \pm \mathrm{SD}$, median value).

${ }^{*} \mathrm{p}<0.05$.

FA, fractional anisotropy; $p$, an independent $t$ test for determination of variances in FA and tract volume between the patient and control groups.

\section{DISCUSSION}

In this study, we investigated injury of the MTT in patients with SAH using DTT. We obtained the following results: (1) the FA value and tract volume in the patient group were significantly lower than those of the control group, (2) MMSE showed a strong ( $r=0.67, p=0.005)$ positive correlation with tract volume without correlation with FA value, (3) in the individual analysis, 16 MTTs of 32 MTTs in 16 patients showed abnormalities of the MTT in terms of the FA value, tract volume or the presence of a reconstructed MTT. As a result, $10(62.5 \%)$ of 16 patients showed abnormality of the MTT in at least one hemisphere. The FA value indicates the degree of directionality of water diffusion and has a range of zero (completely isotropic diffusion) to one (completely anisotropic diffusion). It represents the white matter organisation: in detail, the degree of directionality and integrity of white matter microstructures such as axons, myelin and microtubules. ${ }^{33}$ In contrast, tract volume, the number of voxels in a neural tract, reflects the neural fibres contained within a neural tract. ${ }^{34}$ Therefore, a decrease in the FA or tract volume indicates an injury of the neural tract. Consequently, our results showing that the FA value and tract volume in the patient group were significantly lower than those of the control group indicate an injury of the MTT in the patient group. Regarding the relation between MTT injury and cognition, MMSE showed a strong positive correlation with the tract volume of the MTT without correlation with FA. ${ }^{32}$ These results appear to indicate that the tract volume is more sensitive than the FA value for detection of injury of the MTT. The MMSE is the most widely used tool for screening cognitive dysfunction. ${ }^{35}$ Since this study was conducted retrospectively, we could not employ detailed neuropsychological testing for evaluation of the function of the MTT. Therefore, the conduct of further prospective studies, including specific neurophysiological tests for MTT function, should be encouraged.

The pathogenic mechanism of neural injury following SAH has not been elucidated and few DTI studies have reported on this mechanism. ${ }^{15} 36$ In 2007, Liu et $a l^{36}$ demonstrated that $\mathrm{SAH}$ caused global mild vasogenic oedema in white matter and deep grey matter by measuring the apparent diffusion coefficient value in the subacute stage of $\mathrm{SAH}$, which was undetectable on T2-weighted and diffusion-weighted MR images. In a recent study, Yeo et $a l^{15}$ reported injury of the CST at the midbrain in patients with $\mathrm{SAH}$. This study suggested that the frequent occurrence of SAH in perimesencephalic cisterns could be ascribed to injury of the CST, through mechanical (increased intracranial pressure or direct mass) or chemical mechanisms (a blood clot itself can cause extensive damage). ${ }^{5} 2337$ Considering that the MTT is located in close proximity to a cistern, the MTT in patients with SAH appears to be injured by
Figure 2 The incidence of injury of the mammillothalamic tract. FA, fractional anisotropy; MTT, mammillothalamic tract; TV, tract volume).

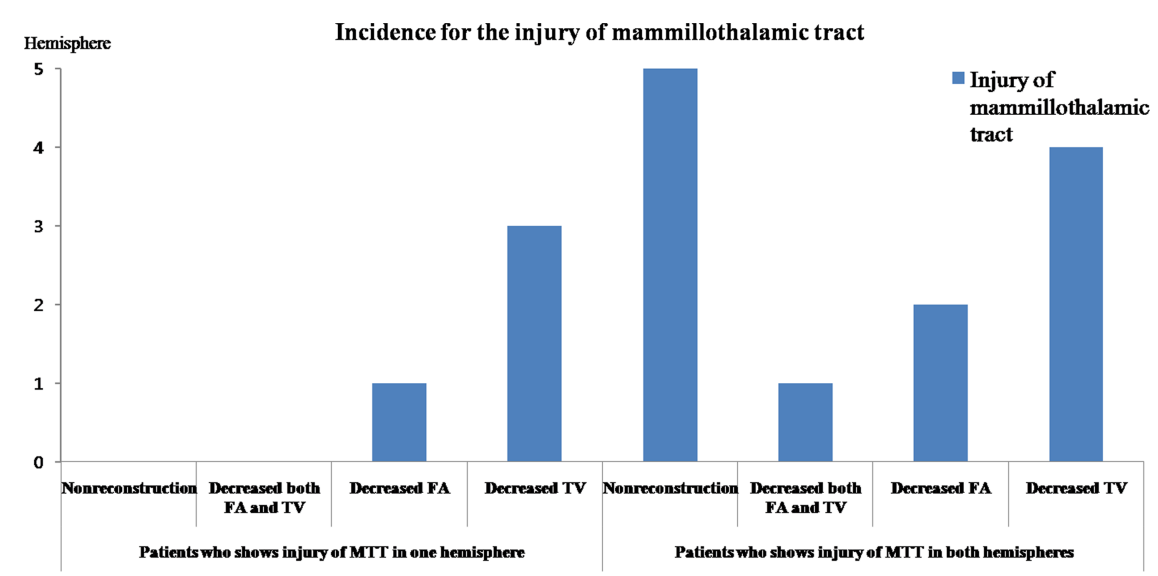


mechanisms similar to those of CST injury at the midbrain. ${ }^{5} 15171823$

As for injury of the MTT, to the best of our knowledge, one study was reported in patients with thalamic haemorrhage. ${ }^{3}$ Therefore, this is the first DTT study on injury of the MTT in patients with SAH. However, several limitations of DTI should be considered. ${ }^{38} 39$ First, DTI is a powerful anatomic imaging tool that can demonstrate gross fibre architecture; however, reflection of all fibres, particularly small fibres, can be difficult. Second, fibre tracking of the MTT might be affected by an artefact, such as an aneurysmal clip. Third, we investigated only injury of the MTT following SAH, even though other tracts such as the fornix, cingulum and thalamocortical tract of the Papez circuit might be also injured. Therefore, conduct of further studies to overcome these limitations would be necessary.

In conclusion, we found injury of the MTT in the patient group, compared with the control group. In addition, in the individual analysis, 16 MTTs (50\%) of 32 hemispheres showed injury of the MTT and $10(62.5 \%)$ of 16 patients showed injury of the MTT in at least one hemisphere in terms of DTT parameters or the presence of a reconstructed MTT.

\section{Author affiliations \\ ${ }^{1}$ Department of Physical Medicine and Rehabilitation, College of Medicine, Yeungnam University, Daegu, Republic of Korea \\ 2Department of Neurosurgery, College of Medicine, Yeungnam University, Daegu, Republic of Korea}

Contributors SHJ was involved in the concept and design of the study, funding, data acquisition and in the development and writing of the manuscript. BYC was involved in the acquisition and analysis of data. SHK and $\mathrm{CHC}$ were involved in the research design and data acquisition. YJJ was involved in the research design and technical support. HGK was involved in data acquisition and in the development, writing and authorisation of the manuscript.

Funding This research was supported by the Basic Science Research Programme through the National Research Foundation of Korea (NRF) funded by the Ministry of Education, Science and Technology (2012R1A1A4A01001873).

\section{Competing interests None.}

Ethics approval This study protocol was approved by the Institutional Review Board of the Yeungnam University Hospital.

Provenance and peer review Not commissioned; externally peer reviewed.

Data sharing statement No additional data are available.

Open Access This is an Open Access article distributed in accordance with the Creative Commons Attribution Non Commercial (CC BY-NC 4.0) license, which permits others to distribute, remix, adapt, build upon this work noncommercially, and license their derivative works on different terms, provided the original work is properly cited and the use is non-commercial. See: http:// creativecommons.org/licenses/by-nc/4.0/

\section{REFERENCES}

1. Aggleton JP, Brown MW. Episodic memory, amnesia, and the hippocampal-anterior thalamic axis. Behav Brain Sci 1999;22:425-44; discussion 44-89.

2. Kwon HG, Hong JH, Jang $\mathrm{SH}$. Mammillothalamic tract in human brain: diffusion tensor tractography study. Neurosci Lett 2010;481:51-3.

3. Jang SH, Lee HD, Kwon HG. Injury of the mammillothalamic tract in patients with thalamic hemorrhage. Front Hum Neurosci 2014;8:259.
4. Jang SH, Jun L, Kwon HG. Mystery case: injuries of neural tracts in the Papez circuit following anterior thalamic infarction. Neurology 2014;82:e178-9.

5. van Gijn J, van Dongen KJ, Vermeulen M, et al. Perimesencephalic hemorrhage: a nonaneurysmal and benign form of subarachnoid hemorrhage. Neurology 1985;35:493-7.

6. Ferrante L, Acqui M, Mastronardi L, et al. Posterior inferior cerebellar artery (PICA) aneurysm presenting with $\mathrm{SAH}$ and contralateral crural monoparesis: a case report. Surg Neurol 1992;38:43-5.

7. Ogden JA, Mee EW, Henning M. A prospective study of impairment of cognition and memory and recovery after subarachnoid hemorrhage. Neurosurgery 1993;33:572-86; discussion 86-7.

8. Ostbye T, Levy AR, Mayo NE. Hospitalization and case-fatality rates for subarachnoid hemorrhage in Canada from 1982 through 1991. The Canadian Collaborative Study Group of Stroke Hospitalizations. Stroke 1997;28:793-8.

9. Powell J, Kitchen N, Heslin J, et al. Psychosocial outcomes at three and nine months after good neurological recovery from aneurysmal subarachnoid haemorrhage: predictors and prognosis. J Neurol Neurosurg Psychiatry 2002;72:772-81.

10. Kreiter KT, Copeland D, Bernardini GL, et al. Predictors of cognitive dysfunction after subarachnoid hemorrhage. Stroke 2002;33: 200-8.

11. Mayer SA, Kreiter KT, Copeland D, et al. Global and domain-specific cognitive impairment and outcome after subarachnoid hemorrhage. Neurology 2002;59:1750-8.

12. Orbo M, Waterloo K, Egge A, et al. Predictors for cognitive impairment one year after surgery for aneurysmal subarachnoid hemorrhage. J Neurol 2008;255:1770-6.

13. Wong GK, Wong R, Mok VC, et al. Clinical study on cognitive dysfunction after spontaneous subarachnoid haemorrhage: patient profiles and relationship to cholinergic dysfunction. Acta Neurochir (Wien) 2009;151:1601-7.

14. Al-Khindi T, Macdonald RL, Schweizer TA. Cognitive and functional outcome after aneurysmal subarachnoid hemorrhage. Stroke 2010;41:e519-36.

15. Yeo SS, Choi BY, Chang $\mathrm{CH}$, et al. Evidence of corticospinal tract injury at midbrain in patients with subarachnoid hemorrhage. Stroke 2012;43:2239-41.

16. Sheldon $\mathrm{S}$, Macdonald $\mathrm{RL}$, Schweizer TA. Free recall memory performance after aneurysmal subarachnoid hemorrhage. J Int Neuropsychol Soc 2012;18:334-42.

17. Smith B. Cerebral pathology in subarachnoid haemorrhage. J Neurol Neurosurg Psychiatry 1963;26:535-9.

18. Grote $\mathrm{E}$, Hassler W. The critical first minutes after subarachnoid hemorrhage. Neurosurgery 1988;22:654-61.

19. Hardemark HG, Almqvist O, Johansson T, et al. S-100 protein in cerebrospinal fluid after aneurysmal subarachnoid haemorrhage: relation to functional outcome, late CT and SPECT changes, and signs of higher cortical dysfunction. Acta Neurochir (Wien) 1989;99:135-44.

20. Jackowski A, Crockard A, Burnstock G, et al. The time course of intracranial pathophysiological changes following experimental subarachnoid haemorrhage in the rat. J Cereb Blood Flow Metab 1990;10:835-49.

21. Germano AF, Dixon CE, d'Avella D, et al. Behavioral deficits following experimental subarachnoid hemorrhage in the rat. J Neurotrauma 1994;11:345-53.

22. Germano A, Caruso G, Caffo M, et al. Does subarachnoid blood extravasation per se induce long-term neuropsychological and cognitive alterations? Acta Neurochir (Wien) 1998;140:805-11 discussion 11-2.

23. Chua $\mathrm{CO}$, Chahboune $\mathrm{H}$, Braun $\mathrm{A}$, et al. Consequences of intraventricular hemorrhage in a rabbit pup model. Stroke 2009;40:3369-77.

24. Hong $\mathrm{JH}$, Choi BY, Chang $\mathrm{CH}$, et al. Injuries of the cingulum and fornix after rupture of an anterior communicating artery aneurysm: a diffusion tensor tractography study. Neurosurgery 2012;70: 819-23.

25. Teasdale GM, Drake CG, Hunt W, et al. A universal subarachnoid hemorrhage scale: report of a committee of the World Federation of Neurosurgical Societies. J Neurol Neurosurg Psychiatry 1988:51:1457.

26. Hijdra A, Brouwers PJ, Vermeulen M, et al. Grading the amount of blood on computed tomograms after subarachnoid hemorrhage. Stroke 1990;21:1156-61.

27. Frontera JA, Claassen J, Schmidt JM, et al. Prediction of symptomatic vasospasm after subarachnoid hemorrhage: the modified fisher scale. Neurosurgery 2006;59:21-7; discussion-7. 
28. Dick JP, Guiloff RJ, Stewart A, et al. Mini-mental state examination in neurological patients. J Neurol Neurosurg Psychiatry 1984;47:496-9.

29. Smith SM, Jenkinson M, Woolrich MW, et al. Advances in functional and structural MR image analysis and implementation as FSL. Neuroimage 2004;23(Suppl 1):S208-19.

30. Behrens TE, Johansen-Berg H, Woolrich MW, et al. Non-invasive mapping of connections between human thalamus and cortex using diffusion imaging. Nat Neurosci 2003:6:750-7.

31. Behrens TE, Berg HJ, Jbabdi S, et al. Probabilistic diffusion tractography with multiple fibre orientations: What can we gain? Neuroimage 2007:34:144-55.

32. Rea LM, Parker RA. Designing and conducting survey research: a comprehensive guide. 3rd edn. San Francisco: Jossey-Bass, 2005.

33. Assaf $\mathrm{Y}$, Pasternak O. Diffusion tensor imaging (DTI)-based white matter mapping in brain research: a review. $J$ Mol Neurosci 2008;34:51-61.
34. Kwak SY, Yeo SS, Choi BY, et al. Corticospinal tract change in the unaffected hemisphere at the early stage of intracerebral hemorrhage: a diffusion tensor tractography study. Eur Neurol 2010;63:149-53.

35. Folstein MF, Folstein SE, McHugh PR. "Mini-mental state". A practical method for grading the cognitive state of patients for the clinician. J Psychiatr Res 1975;12:189-98.

36. Liu Y, Soppi V, Mustonen T, et al. Subarachnoid hemorrhage in the subacute stage: elevated apparent diffusion coefficient in normalappearing brain tissue after treatment. Radiology 2007;242:518-25.

37. Jang SH. Somatotopic arrangement and location of the corticospinal tract in the brainstem of the human brain. Yonsei Med J 2011;52:553-7.

38. Yamada K, Sakai K, Akazawa K, et al. MR tractography: a review of its clinical applications. Magn Reson Med Sci 2009;8:165-74.

39. Lee SK, Kim DI, Kim J, et al. Diffusion-tensor MR imaging and fiber tractography: a new method of describing aberrant fiber connections in developmental CNS anomalies. Radiographics 2005;25:53-65; discussion 6-8. 